\title{
Relación entre satisfacción familiar y rendimiento académico en estudiantes de psicología de la Universidad Pontificia Bolivariana Bucaramanga.
}

\author{
Relationship between family satisfaction and academic achievement in students of psychology at the \\ University Pontificia Bolivariana Bucaramanga.
}

\author{
Gustavo Villamizar Acevedo ${ }^{1}$, Jenny Paola Galvis Mateus², Marcela Jiménez Plata ${ }^{3}$
}

\section{RESUMEN}

Esta investigación buscó establecer la relación entre satisfacción familiar y rendimiento académico en 95 estudiantes de II, III y IV semestre de psicología de la Universidad Pontificia Bolivariana de Bucaramanga. Para su desarrollo se utilizó un método de corte cuantitativo, no experimental, de diseño correlacional. La satisfacción familiar se identificó por medio de la Escala de Satisfacción Familiar por Adjetivos (ESFA), el promedio académico por la suma de las notas de las asignaturas vistas sobre el número de materias. Los resultados obtenidos en las pruebas mostraron satisfacción familiar y promedio académico regular. Una vez obtenido los resultados en la ESFA e identificado el promedio académico se aplicó la $r$ de Pearson para encontrar el coeficiente de correlación, para ello se utilizó el SPSS. Los resultados mostraron la inexistencia de correlación entre esas dos variables, tanto en la totalidad de los participantes como por nivel de formación.

Palabras Claves: Satisfacción familiar, funcionamiento familiar, rendimiento académico, estudiantes universitarios.

\section{SUMMARY}

This research sought to establish the existence of relationship between family satisfaction and academic achievement in 95 students of II, III and IV semester of psychology at the Universidad Pontificia Bolivariana. For its development we used a quantitative method of cutting, non-experimental correlated design. Family satisfaction identified through the Family Satisfaction Scale with Adjectives (ESFA), the grade point average in the amount of notes subject views the number. The test results showed family satisfaction, tendency to dysfunctional families and regular academic average. After obtaining the results in the ESFA and identified academic average was performed to find Pearson's correlation coefficient, for it was used SPSS. The results showed no correlation between these two variables, in all the participants and by level of education

Keywords: Family satisfaction, family functioning, academic performance, college students.

1 Psicólogo. Dr. en Educación. Profesor de la Facultad de Psicología de la Universidad Pontificia Bolivariana Bucaramanga (Colombia) gustavo.villamizar@upb.edu.com

2 Psicóloga. la Universidad Pontificia Bolivariana Bucaramanga (Colombia) jepagama20@hotmail.com

3 Psicóloga. la Universidad Pontificia Bolivariana Bucaramanga (Colombia) jimenezmarcela169@gmail.com 


\section{INTRODUCCIÓN}

Una de las grandes preocupaciones en el mundo académico actual tiene que ver con el rendimiento académico y la identificación de los factores que se encuentran asociados, tal hecho se expresa en el interés por detectar las diversas variables que influyen en el logro de los estudiantes, las cuales se ha corroborado son multicausales.

La Universidad Pontificia Bolivariana Bucaramanga (UPB) no ha sido ajena a dicho interés, situación que se manifiesta en la creación de diversos programas con el objetivo de promover un elevado nivel de rendimiento para garantizar la permanencia de sus estudiantes. Entre ellos se encuentran el Programa de Acompañamiento Académico (PAC) que ofrece atención a estudiantes con dificultades académicas, el Programa Integrado de Prevención de Deserción Académica (PAD) dirigido a los estudiantes de primer semestre de todas las carreras de la Universidad y el Programa de Tutorías Académicas que busca "favorecer el desempeño académico de los estudiantes que se vinculan al programa de tutorías, previniendo la deserción estudiantil y propiciando espacios para el mejoramiento de nivel académico de los estudiantes de todas las carreras y semestres de la Universidad" (Martínez, 2011, p. 20).

Como se aprecia los diversos programas mencionados se han centrado en aspectos cognitivos dejando de lado otro tipo de variables como las relacionadas con factores familiares, a pesar que en unos de los programas del PAC, el de Fortalecimiento Académico, se reconoce la importancia que la familia juega en el proceso formativo, por ello ahí se planteó como uno de sus objetivos integrar la familia.

El papel que la familia cumple en el rendimiento académico de los estudiantes universitarios ha sido reconocido por varias investigaciones como las realizadas por Torres y Rodríguez (2006) quienes hallaron relación entre el apoyo familiar percibido por los estudiantes y rendimiento académico. Por su parte Orozco y Velásquez (2010) encontraron riesgo en el rendimiento de los estudiantes pertenecientes a familias con problemas afectivos. Como se aprecia estasinvestigaciones se centraron en el estudio de variables relacionadas con la percepción de apoyo, clima familiar, ambiente psicoafectivo.

Teniendo en cuenta que las tres variables mencionadas se pueden agrupar en una categoría mayor: satisfacción familiar, y tomando en cuenta el reconocimiento explícito, por parte de algunos de los programas de Bienestar Universitario, acerca del papel que la familia juega en el logro académico de los estudiantes; y que al respecto, en la Universidad Pontificia Bolivariana Bucaramanga no se ha realizado estudios que hayan determinado la relación entre satisfacción familiar y rendimiento académico, es que se plantea el siguiente problema de investigación: ¿Existe relación entre rendimiento académico y satisfacción familiar en estudiantes que cursan de segundo a cuarto semestre de Psicología en la Universidad Pontificia Bolivariana Bucaramanga?. El objetivo que se planteó fue: Identificar la relación entre satisfacción familiar y rendimiento académico en estudiantes de segundo al cuarto semestre de Psicología de la Universidad Pontificia Bolivariana Bucaramanga.

Teniendo en cuenta que esta investigación gira alrededor de los conceptos satisfacción familiar y rendimiento académico, pero que para hablar de satisfacción familiar es necesario hablar de familia, el marco conceptual tiene en cuenta dos apartados. En el primero se abordan los conceptos centrales: familia, satisfacción familiar y rendimiento académico. En el segundo se presentan diversas investigaciones relacionadas con la temática, para ello se parte de los trabajos investigativos realizados en la UPB Bucaramanga.

Respecto a familia, se puede asegurar que de todas las instituciones humanas, si hay una que tenga carácter general es la familia, por ello siguiendo a Murdock, citado por Rodríguez (2001) se puede decir que es una organización presente en todas las sociedades humanas.

Sobre el origen del término familia actualmente no hay claridad, para el más prestigioso de los etimologista de la lengua castellana, Corominas (2003), proviene del latín famulus cuyo significados son esclavos o sirvientes. Él afirma que en un comienzo el término hacía referencia al conjunto de esclavos y criados propiedad de un solo hombre. Para otros autores proviene del latín fames que significa hambre, que puede referirse tanto de alimento como a lo sexual, ya que en este grupo se sacian estos dos tipos de necesidades (Nizama, 2009).

El hecho de que los términos famulus y fames sean latinos permite suponer que la palabra nació en Roma, Según Engels (1976) se la inventaron los romanos para designar un nuevo organismo social, cuyo jefe tenía bajo su poder a la mujer, a los hijos y a cierto número de esclavos, con la patria potestad romana y el derecho de vida y muerte sobre todos ellos (p. 55). 
Ackerman (1958) presenta una descripción de la familia haciendo ver las contradicciones en las cuales se mueve. Sostiene que la familia es el nombre de una institución tan antigua como la misma especie humana. Es una entidad paradójica y evasiva. Asume muchas apariencias. Es la misma en todas partes y sin embargo no es nunca la misma. A través del tiempo ha permanecido igual, $\mathrm{y}$ sin embargo nunca ha permanecido igual (p. 35).

Según Levi-Strauss, citado por Estupiñan y Córdoba (1992) la familia es producto de una ley natural que se caracteriza por los siguientes aspectos: a) La familia tiene su origen en el matrimonio. b) Incluye al marido, la mujer y los hijos nacidos de esa unión, formando un núcleo al que eventualmente pueden agregarse otros parientes. c) Los miembros de la familia están unidos entre sí por lazos jurídicos; por derechos y obligaciones de naturaleza económica y religiosa, entre otros; por una red precisa de derechos y prohibiciones sexuales, y por un conjunto variable y diversificado de sentimientos, tales como afecto, respeto, temor. (p. 8).

Alrededor del término familia se han creado múltiples conceptos, por ejemplo se presentan las siguientes: Pérez $(2008$, p. 1) la considera un grupo en la cual quienes la conforman "se cohesionan, se quieren, se vinculan y así se ayudan recíprocamente a crecer vitalmente, a vivir como personas en todas sus dimensiones: cognitiva, afectiva, relacional".

Según Galvis (2001, p.73) es un "conjunto de personas unidas por lazos de parentesco, pero también por vínculos de amor, solidaridad, lealtad y respeto a la dignidad de sus componentes".

Para Minuchin (1984) es un grupo el cual sus integrantes desarrollan sentimientos de identidad e interdependencia. De identidad en la medida que la persona asume pertenecer a ese grupo, a ser parte de él. De interdependencia debido a que se da cuenta que ella está conformada por diferentes subsistemas y que 'él pertenece a uno de ellos.

Según la Constitución Política de Colombia (2010, p.12) "la familia es el núcleo social de la sociedad. Se constituye por vínculos naturales o jurídicos, por la decisión libre de un hombre y una mujer de contraer matrimonio o por la voluntad expresa de conformarlo".

Por otra parte, se puede ver a la familia como un sistema, es decir: "La familia es un conjunto organizado e interdependiente de personas en constante interacción, que se regula por unas reglas y por funciones dinámicas que existen entre sí y con el exterior" (Minuchín, 1984).

Espinal, Gimeno y González (2003) consideran que a partir del enfoque sistémico los estudios de familia se basan, no tanto en los rasgos de personalidad de sus miembros, como características estables temporal y situacionalmente, sino más bien en el conocimiento de la familia, como un grupo con una identidad propia y como escenario en el que tienen lugar un amplio entramado de relaciones.

Para efectos de la presente investigación se consideró pertinente asumir la definición de Hernández (1992) quien considera la familia como un sistema, en la medida en que está constituida por una red de relaciones; es natural, porque responde a necesidades biológicas y psicológicas, inherentes a la supervivencia humana; y tiene características propias, en cuanto que no hay ninguna otra instancia social que hasta ahora haya logrado reemplazarla como fuente de satisfacción de las necesidades psicoafectivas tempranas de todo ser humano (p. 26).

Esta visión de la familia sigue los principios establecido por Bertalanffy, quien según Soria (2010) definió sistema como una serie de elementos interrelacionados con un objetivo común, que se afectan unos a otros, y la característica que los une es la composición que tienen, es decir, la totalidad, la cual no es sólo la suma de las partes, sino también la relación entre ellas, además, cada elemento tiene una función e interactúan entre ellos (p. 89).

Desde esta perspectiva la familia, es vista como un sistema abierto, en constante interacción, compuesto por subsistemas, como el conyugal, el fraterno y el parento-filial. Cada integrante de la familia también es considerado un sistema, compuesto a su vez de subsistemas. Pero ambos, persona y familia, se encuentran ligados a un suprasistema, que puede ser inmediato (barrio) o más amplio, la sociedad en general.

Estas propiedades pertinentes para la presente investigación son desarrolladas por Membrillo y Ocampo (2008) de la siguiente forma: Teniendo en cuenta que los sistemas están conformados por subsistemas, al considerar la familia como sistema conlleva a aceptar que de ella es parte un conjunto organizado de subsistemas, cada uno de los cuales tiene 
su propia denominación de acuerdo a las tareas que realiza. Ellos son el subsistema parental, el subsistema conyugal, el subsistema fraternal y otros. Un miembro de la familia puede pertenecer a varios subsistemas, por ejemplo puede ser esposo y padre. Los miembros que conforman un sistema se encuentran ligados, en esa medida algo que afecte un miembro afecta la totalidad del sistema.

En los sistemas el todo es más que la suma de las partes, en esa medida lo que caracteriza una familia no debe ser vista como la totalidad de la suma de los comportamientos de cada integrante sino el producto de las interrelaciones que se dan entre sus miembros y de sus mutuas influencias: La retroalimentación es permanente y cumple el papel de autorregulador de la conducta, esto se evidencia al darse cuenta como el comportamiento de uno de sus miembros genera reacciones en los otros, esta acción-reacción les permite a sus miembros realizar ajustes que incidan en su comportamiento futuro.

Aparte de los cuatro elementos mencionados existe un conjunto de interacciones que busca propiciar el mantenimiento del sistema familiar, ellos son: la comunicación, la organización y gestión de las normas y la postura frente al desarrollo de los roles (Parra, Gomaris \& Sánchez, 2011).

De estos elementos, en la investigación, se resalta el papel de la comunicación. Esta permite a la familia organizar las funciones, reglas, tareas, normas, conformando la estructura que mantiene el sistema familiar en sí (Palomar \& Suarez, 1993). El papel de la comunicación familiar en el desempeño académico fue resaltado por Corsi (2003) quien señaló que un ambiente de disputas, reclamos y recriminaciones afecta la calidad en las actividades estudiantiles.

Otro aspecto a analizar tiene que ver con la satisfacción familiar. Cuando las personas hablan acerca de satisfacción, los criterios de evaluación que utilizan son de carácter subjetivo, personal, depende de la percepción que tengan de la situación. Cuando se trata de satisfacción familiar corresponde, según Velásquez, Vaiz y Luiz (2009) a una "respuesta afectiva resultante de la interacción entre los miembros de la familia, en el cual, el sujeto encuentra relaciones familiares positivas, la existencia de un clima familiar agradable permite disfrutar y compartir momentos positivos entre los miembros de la familia" (p. 13).

Sobrino (2008) entiende la satisfacción familiar como un proceso fenomenológico en el cual los integrantes de una familia la perciben y valoran a partir de los niveles comunicacionales que se da entre los que la conforman, el grado de cohesión entre los miembros y el índice de adaptabilidad familiar de los miembros entre sí y con su entorno.

A partir del análisis de las formas de interacción que se dan en las familias, Jackson citado por Sobrino (2008) clasificó la satisfacción en cuatro tipos: satisfacción estable, satisfacción inestable, insatisfactoria estable e insatisfactoria inestable. Las características de cada una son las siguientes: Satisfacción estable: en esta familia los elementos relacionales son lo suficientemente claros como para saber quién tiene el control y toma decisiones. En este grupo a pesar de la existencia de dificultades rápidamente se retorna a la normalidad. Satisfacción inestable: se presenta al comienzo de una relación, en ellas la pareja va reelaborando la relación hasta lograr acordar sus responsabilidades. Insatisfactoria estable: en este caso la pareja no discute sobre quién está a cargo de la relación. Se tiende a dejar el control del funcionamiento familiar, en elementos externos que puede ser en reglas sociales o religiosas: Insatisfactoria inestable: aquí la pareja nunca llega a un acuerdo sobre cómo se van a manejar ciertas aéreas o quién debe tomar la iniciativa en ciertos momentos, esta situación lleva a que los momentos de estabilidad sean muy breves y los de inestabilidad prolongada.

Para esta investigación se asume satisfacción familiar de acuerdo a los postulados de Barraca y López-Yarto (1997), quienes la ven como el producto de diversos sentimientos que se generan en las personas al compartir con su familia. Su origen tiene que ver con las interacciones que se producen entre un individuo y los integrantes de su grupo familiar. Según los criterios establecidos en la Escala de Satisfacción Familiar por Adjetivos (ESFA), una persona con un grado de satisfacción alto es aquella que está convencida que en su familia las interacciones positivas son mayores que las negativas.

En relación con rendimiento académico, tratar de definirlo no es una tarea sencilla, debido a que existen múltiples criterios, González; citada por Narváez (1999) encontró que los investigadores utilizan cinco formas para determinarlo: 1) Como promoción o flujo de estudiantes, para ello comparan el número de estudiantes que ingresan con el de los que continúan. 2) Como el promedio de notas obtenidas por el estudiante. 3) Como variable dicotómica: no repitencia 
Vs. Repitencia. 4) Como el promedio de notas ponderado por la relación de créditos aprobados sobre créditos cursados. 5) Como el rendimiento tomando en cuenta notas, relación entre materias aprobadas sobres cursadas y tiempo de graduación.

Narváez (1999) ve el rendimiento académico como un conjunto de números que se toman como la huella de aciertos y fracasos en el recorrido escolar.

Edel(2003) lo define como un constructo susceptible de adoptar valores cuantitativos y cualitativos, a través de los cuales existe una aproximación a la evidencia y dimensión del perfil de habilidades, conocimientos, actitudes y valores desarrollados por el alumno en el proceso de enseñanza aprendizaje.

Para Pizarro, citado por Reyes (2003) es una medida de las capacidades correspondientes o indicativas que manifiestan en forma estimativa, lo que una persona ha aprendido como consecuencia de un proceso de instrucción o formación.

Para esta investigación se asume como el promedio de notas ponderado por la relación de créditos aprobados sobre créditos cursados.

En lo que atañe a factores relacionados con rendimiento académico, diversos investigadores han mostrado que está influenciado por múltiples factores algunos son de carácter personal, otros están asociados a situaciones ambientales, algunos más a circunstancias culturales. Al respecto, Tinto (1989), plantea que el rendimiento académico está influenciado por antecedentes familiares, características individuales, antecedentes educativos y compromiso, por parte del estudiante, por alcanzar las metas que se planteó como proyecto de vida.

Romo y Fresan, y Rodríguez, referidos en Aldana, Pérez y Rodríguez (2010) identificaron cuatro factores influyentes en el logro académico: fisiológicos, tienen que ver con el estudiante; pedagógicos, relacionados con la calidad de la enseñanza y el aprendizaje, se consideran variables como tamaño del grupo, métodos y estrategias de estudio, calidad del docente; psicológicos, asociados a variables de personalidad, motivacionales, actitudinales y afectivas de los alumnos, expectativas, vocación, satisfacción; y sociológicas, que incluyen las condiciones socioeconómicas, familiares y condiciones laborales.

Según Enríquez (2008) el rendimiento académico es multicausal en la medida que está acompañado de factores tanto internos (hábitos y herramientas de estudio y motivación e interés en el estudio) como externos (contexto social y familiar) en el cual se desenvuelve y se da la cotidianidad del adolescente.

Por otra parte, Artunduaga (2008) al referirse sobre las variables influyentes en el rendimiento académico de los estudiantes universitarios, las divide en dos grandes factores: los contextuales; en donde se encuentran las variables socioculturales, institucionales y pedagógicas, y; los personales, en donde se ubican las variables demográficas, cognoscitivas y actitudinales. Producto de sus investigaciones este autor ha encontrado que el medio sociocultural en que vive el estudiante, el nivel educativo de los padres, el grado de expectativa de los padres sobre los hijos, así como el grado de integración y participación social de los estudiantes tiene una gran influencia en el rendimiento académico. De igual forma, las variables relacionadas con el plantel como las políticas educativas, el tamaño y tipo de institución y las que tienen que ver con niveles de participación y gestión.

La identificación de esta multiplicidad de factores llevó a establecer dos grandes categorías: endógenas y exógenas. En la tabla 1 se sintetizan las características de cada una de ellas.

En relación a factores exógenos, la familia juega un papel fundamental, pero no sólo en lo relacionado con variables sociales, también resaltan las que tienen que ver con el clima familiar, con la dinámica que se mueve en el hogar.

En un estudio realizado en Guadalajara, PazNavarro, Rodríguez y Martínez (2009) evaluaron el funcionamiento familiar de 65 bachilleres con bajo rendimiento escolar (BRE) y lo compararon con un grupo de 90 estudiantes con rendimiento escolar promedio (REP) de una Preparatoria de la Universidad de Guadalajara. Los resultados obtenidos permitieron evidenciar que el $54 \%$ de las familias de alumnos con BRE eran completas, comparadas con el $73 \%$ de familias con alumnos con REP.

Los alumnos con bajo rendimiento académico obtuvieron mayores puntajes en la valoración del poder, dinero, sexo y satisfacción con la vida. Los alumnos con rendimiento académico promedio obtuvieron mayores puntajes en participación en la solución de problemas, comunicación padre-hijo, redefinición de los problemas, apoyo, menores síntomas y problemas 
Tabla 1. Características de los factores que intervienen en el rendimiento académico.

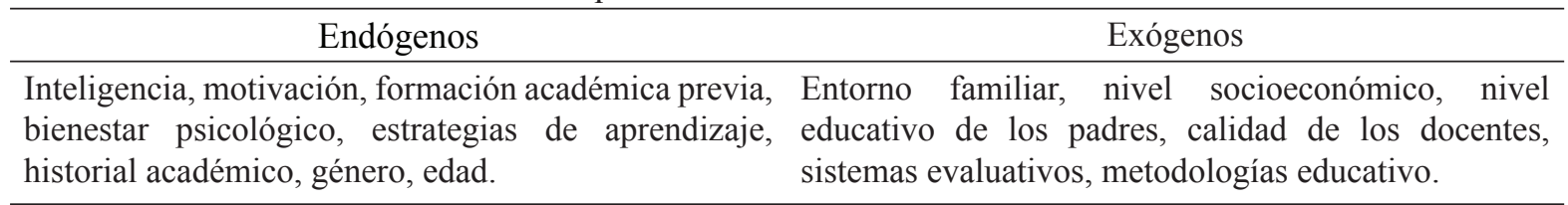

Fuente: elaborada por los autores.

y mayor educación de ambos padres. Otro aspecto relevante es que las familias con hijos de BRE tuvieron mayor nivel de vulnerabilidad en su funcionamiento familiar comparadas con las familias de alumnos con REP.

Esta diferencia resulta coincidente con lo que menciona Fishman, citado por Andrade y Montalvo $(2006,88)$ quien dice: "todos los cambios que sobrevienen en los sistemas sociales de los que participa el adolescente, los que más lo afectan son los que se producen en su familia". Los autores mencionan que el adolescente resulta sumamente vulnerable a los cambios contemporáneos dentro de la estructura familiar. Ellos también encontraron que los alumnos que tienen familias diferentes a las consideradas como tradicionales, poseen características que influyen en el bajo rendimiento de los adolescentes, tal vez el tipo de sociedad conservadora donde se encuentra esta preparatoria todavía hace que el vivir en una familia reconstruida o monoparental genere problemas de tipo social que se ven reflejados en la actividad escolar de los adolescentes.

Martínez, Sanz y Cosgaya (2005) investigaron acerca del papel de las relaciones familiares en el rendimiento escolar y el bienestar psicológico en estudiantes de enseñanza secundaria, para ello se analizaron variables como percepción de los hijos del conflicto interparental (conflicto matrimonial, relaciones parentales) y satisfacción familiar. En la primera variable (conflicto matrimonial) entendida como la relación entre el grado de conflicto percibido entre los padres y el rendimiento de los hijos, encontraron que cuanto mayor es el conflicto percibido, peor es el rendimiento escolar, en todas las dimensiones estudiadas. Es decir, cuando el conflicto es mayor, disminuye el logro académico (las notas son peores y existe más tendencia a repetir curso), el comportamiento académico también empeora (tendencia a distraerse, a hablar en clase, a no realizar las tareas...) y aumentan las dificultades, para concentrarse y el cansancio a la hora de realizar las tareas es mayor.
Al analizar las dimensiones del conflicto encontraron que, cuando el hijo percibe que los conflictos entre sus padres se deben a cuestiones relacionadas con él/ella o bien se siente culpable por tales conflictos, aumentan sus problemas escolares. De igual manera, cuando el conflicto es intenso, se percibe que durará en el tiempo, que es muy frecuente, y que a menudo los temas de discusión quedan sin resolver, el impacto es mayor. Y finalmente, cuando los hijos se implican en el conflicto con sus padres (triangulación) el rendimiento es menor.

En una segunda variable, se encontraron correlaciones positivas entre las relaciones parentales y el rendimiento escolar. Esto ocurre tanto en relación con la madre como con el padre. La conclusión es clara, cuanto mayor es el amor percibido por los hijos en las relaciones con los padres y menor el control y la hostilidad, se observa mejor rendimiento. Las variables "amor y hostilidad" las que mayores correlaciones presentan. La tercera variable, la satisfacción familiar quizá sea la variable más vinculada con el rendimiento escolar, ya que los alumnos que señalaron estar más satisfechos con su familia, fueron quienes mejor rendimiento demostraron.

De manera general se puede señalar que las variables familiares, sobre todo, el conflicto matrimonial, tienen un gran impacto en el rendimiento escolar. Los intereses del estudiante están reabsorbidos por los conflictos familiares, lo que disminuye su capacidad y motivación para interesarse y enfrentarse con los problemas y dificultades de todo tipo, entre ellos los escolares.

Garbanzo (2007) encontró que las relaciones con el padre, la madre y el ambiente del estudiante, influye significativamente en la vida académica. De esta manera, un ambiente familiar adecuado, marcado por el compromiso incide en el desempeño académico, lo mismo que una convivencia familiar democrática entre padre e hijos. Los resultados de éste trabajo arrojaron asociación entre convivencia familiar democrática y desempeño académico, no presentando los mismos resultados para los estudiantes que viven 
con familiares autoritarios o indiferentes. Afín a los resultados encontrados por Garbanzo (2007), GarcíaCruz, Guzmán y Martínez (2006) encontraron que entre las variables influyentes en las notas o calificaciones de los estudiantes, el factor que más prevalece es la comunicación familiar.

En relación a esto, Contreras, Caballero, Palacio y Pérez (2008) entrevistaron a un grupo de jóvenes, que presentaban bajo rendimiento debido a múltiples factores, entre ellos se mencionan los motivacionales originados en problemas familiares. De la totalidad de los participantes el 18\% consideró que la relación con sus padres era mala, el $21 \%$ no manifestó nada al respecto.

Estudios del Banco Mundial, según Garbanzo (2007) han demostrado que el $60 \%$ de los resultados académicos se pueden explicar por factores externos al entorno educativo donde el clima del hogar y la cantidad de años de estudio de los padres, se encuentran entre los factores con mayor peso en el rendimiento académico. Relacionado con esto Pérez y Castejón, así como, Andrade, Miranda y Frías, citados por García-Cruz, Guzmán y Martínez (2006) notaron en sus trabajos investigativos una gran influencia entre rendimiento académico y dinámica familiar. Del mismo modo, Gómez, citado por García-Cruz, Guzmán y Martínez (2006) concluyó que existe relación entre el clima familiar en el cual el estudiante ha vivido y el éxito escolar.

García (2005) estudiando un conjunto de variables relacionadas con el rendimiento académico, objetó la inexistencia de correlaciones significativas entre las calificaciones y el clima social de la familia; resultados que afirman difieren de los presentados por Guerra (1993) quien halló relación entre el bajo rendimiento con hogares desorganizados.

En la UPB se ha abordado esta temática tal como lo muestra una serie de trabajos que se han realizado. Cely y Trouchon (1999) estudiaron las características personales y familiares de los estudiantes que ingresaron al primer semestre de 1998, a partir del análisis de 46 entrevistas realizadas con padres de familia, notaron que el $50 \%$ de ellos conciben las relaciones familiares fundamentadas en la amistad y la confianza, un $30 \%$ considera esas relaciones como buenas, un $10 \%$ las ve como normales, otro $10 \%$ las percibe como regulares. Las investigadoras agruparon las respuestas en dos grandes categorías: buenas y difíciles, los resultados mostraron que el $80 \%$ de los padres consideran que las relaciones que llevan con sus hijos son buenas y el $20 \%$ restante las considera difíciles.

Posteriormente, Cely y Ojeda (2003) replicaron esta investigación con una muestra de personas admitidas a la universidad en el 2002. Los resultados mostraron que el $54 \%$ de los padres consideran que la relación que tienen con sus hijos es buena, el 13\% la ve como normal, el $7 \%$ considera que sus hijos ya son personas independientes y el 1.5\% los conciben como distantes. Se nota una disminución en la percepción de los padres acerca de su relación con sus hijos, a pesar del corto tiempo de diferencias, esto lo que muestra es que los cambios generacionales son cada vez más cercanos en el tiempo.

Higuera y Martínez (2002) al realizar su trabajo de grado con estudiantes admitidos en primer semestre del 2002 en la UPB, hallaron que el $80 \%$ de los encuestados mostraron una actitud positiva hacia la unión familiar, lo que muestra la importancia que los estudiantes le dan a la conformación de un grupo familiar afectivamente sólido y a los niveles de comunicación adecuadas. La existencia del sentimiento de unión familiar les brinda a los estudiantes estabilidad emocional y consideran que esto les ayuda en la formación.

Hernández y Echavarría (2007) analizaron las características de la estructura familiar de los estudiantes de la Escuelas de Ingeniería y Administración y la de Derecho y Ciencias Políticas. Los investigadores encontraron que más del $60 \%$ de las familias son de tipo nuclear y que tienen entre dos $\mathrm{y}$ tres hijos por promedio.

Villamizar y Romero (2011), al investigar sobre factores relacionados con rendimiento académico en estudiantes de primer semestre del 2009 de psicología, encontraron que los estudiantes que viven en familias nucleares tienen mejor rendimiento académico.

Como se aprecia, estas investigaciones no abordaron las relaciones entre la estructura familiar, las relaciones que se dan entre sus integrantes y el rendimiento académico; ellas indagaron sobre la conformación predominante, encontrando que es la nuclear, que los padres resaltan la importancia de la unión familiar y el valor que en ella juega la comunicación y la estabilidad emocional. 


\section{METODOLOGÍA.}

La investigación fue descriptiva de tipo correlacional, porque se buscó establecer la relación entre las variables continuas satisfacción familiar y rendimiento académico.

Para su desarrollo empírico se contó con la participación de la totalidad de los estudiantes de Psicología de la UPB matriculados en segundo, tercero y cuarto semestre, previa aceptación. Para ello se les entregó un consentimiento informado, el cual una vez leído y aprobado los habilitaba para hacer parte de la investigación. A la fecha de la realización de la investigación se encontraban matriculados 100 estudiantes, de los cuales 95 aceptaron participar, ver tabla 2.

En relación con los instrumentos de medida, se aplicó las Escala de Satisfacción Familiar por Adjetivos (ESFA). Para conocer el promedio académico de cada estudiante se diseñó una matriz donde se consignaban el nombre de la asignatura y la nota alcanzada al terminar el curso.

La Escala de Satisfacción Familiar por Adjetivos (ESFA) fue construida por Barraca y López-Yarto (2003), con una estructura propia del diferencial semántico, está compuesta por 27 ítems cada uno formado por una pareja de adjetivos antónimos, sus preguntas buscan evocar una respuesta preferentemente afectiva. Tiene como objetivo evaluar la satisfacción familiar, puede ser respondida por mayores de 16 años, en forma individual o colectiva.

El grado de fiabilidad de la escala es significativamente alto, medido a través del Alfa de Cronbach el resultado es de .9758 . En lo que atañe a su validez, el análisis factorial realizada la justificó, la validez de criterio mediante la comparación de las puntuaciones con otros dos instrumentos de medida de la satisfacción familiar (FamilySatisfaction y FamilySatisfactionScale), es satisfactoria (entre 0,64 y $0,78)$. Finalmente, un estudio realizado con 16 sujetos clínicos, cuyas puntuaciones fueron comparadas con una muestra normal de similar tamaño, evidenció la capacidad discriminativa de la escala.

\section{Estructura de la prueba.}

Consta de 27 adjetivos bipolares, con seis opciones de respuesta según el modelo Likert, tal y como se ilustra en la tabla 3. El valor dado a la respuesta, según lo estipula el manual, está en relación con la cercanía al adjetivo. La conversión de los puntajes, permite identificar cuatro rangos de satisfacción:

- Muy baja satisfacción familiar

- Baja satisfacción familiar

- Moderada satisfacción familiar

- Alta satisfacción familiar

Para la calificación se tiene en cuenta el siguiente criterio: puntuaciones por encima de la media señalan experiencia satisfactoria con la familia, considerándose como altas todas las que estén encima del centil 70 y bajas las que se encuentren por debajo del centil 30 .

Para efectos de la calificación se tuvo en cuenta la dirección de los adjetivos. Se consideraron positivos los que van de una situación generadora de satisfacción a una de insatisfacción como los siguientes: 1, 3, 4, 6, $9,10,13,17,20,22,24$, y 26. Como negativos los que van de una situación generadora de insatisfacción a satisfacción, como tales se valoran los restantes. Para obtener el promedio académico los autores diseñaron una matriz de dos entradas, en una este muestra las asignaturas que los estudiantes deben haber cursado desde primer semestre hasta cuarto, la otra corresponde a la nota obtenida por el estudiante en dicha asignatura, tal como se muestra en la tabla 4.

Tabla 2. Número de estudiantes matriculados en segundo, tercero y cuarto en Psicología

\begin{tabular}{ccc}
\hline Semestre & Estudiantes & Porcentaje \\
\hline Segundo & 46 & $48 \%$ \\
Tercero & 21 & $22 \%$ \\
Cuarto & 28 & $30 \%$ \\
Total & 95 & $100 \%$ \\
\hline
\end{tabular}

Tabla 3. Calificativos escala de satisfacción familiar por adjetivos

\begin{tabular}{cccccc}
\hline Totalmente & Bastante & Algo & Algo & Bastante & Totalmente \\
\hline 6 & 5 & 4 & 3 & 2 & 1 \\
\hline
\end{tabular}


Para recoger información personal se elaboró una encuesta donde se indagó sobre la edad de los participantes, lugar de procedencia, estrato social del barrio donde reside y las personas con las cuales vive, también se le solicitó una apreciación sobre la relación que lleva con su familia.

\section{Procedimiento.}

Esta investigación se desarrolló en las siguientes fases: definición de la situación problema, selección de la metodología, de los instrumentos, del escenario y de los participantes, trabajo de campo y análisis de la información.

A continuación se presenta una breve descripción de cada una:

Fase 1. Definición de la situación problema: es el resultado de un proceso de trabajo previo de las investigadoras, lecturas, investigaciones en la línea de satisfacción familiar y rendimiento académico. A esta fase tambien corresponde la construcción del marco teórico.

Fase 2. Selección de la metodología y de los instrumentos: esta es una selección del diseño y de las técnicas para recopilar información, establecidas las técnicas se procedió a seleccionar los instrumentos y los participantes.
Fase 3. Selección de los participantes: en este momento se definió el lugar donde se realizó la investigación así como las personas que intervinieron en ella como participantes. Se seleccionó como muestra de estudio a los estudiantes matriculados en los semestres II, III y IV.

Fase 4. Trabajo de campo: se aplicaron los diferentes instrumentos, previamente se solicitó a algunos docentes de los cursos, su colaboración para poder desarrollar esta actividad antes de la terminar su clase. Se les explicó a los participantes el objetivo de la investigación, se leyeron las instrucciones para responder las pruebas.

Fase 5. Análisis de la información: este proceso comenzó con puntuación de los resultados de las pruebas y la obtención del promedio de notas de cada estudiante, se siguió con la calificación de las pruebas. Posteriormente se realizó la correlación entre los resultados arrojados por la ESFA y el rendimiento académico utilizando para ello el SPSS versión 17, una vez realizado este procedimiento se continuó con el análisis e interpretación de los resultados.

\section{RESULTADOS}

Inicialmentesepresentalos datossociodemográficos de los estudiantes, luego su percepción sobre la relación familiar, después los datos correspondientes

Tabla 4. Matriz de promedio académico.

\begin{tabular}{cc}
\hline Asignaturas & Notas \\
\hline 1 & \\
2 & \\
3 & Sumatoria de notas/número de asignaturas \\
\hline
\end{tabular}

Tabla 5. Distribución de los participantes por género.

\begin{tabular}{ccc}
\hline Género & Participantes & Promedio \\
\hline Masculino & 19 & $20 \%$ \\
Femenino & 76 & $80 \%$ \\
\hline
\end{tabular}

Tabla 6. Distribución estudiantes por rango de edad.

\begin{tabular}{|c|c|c|c|c|c|}
\hline \multirow{2}{*}{ Semestre } & \multicolumn{2}{|c|}{ Edad } & \multicolumn{2}{|c|}{ Edad } & \multirow{2}{*}{ Total } \\
\hline & 16 a 21 & Porcentaje & 22 a 28 & Porcentaje & \\
\hline II & 45 & $48 \%$ & 1 & $1 \%$ & 46 \\
\hline III & 17 & $18 \%$ & 4 & $4 \%$ & 21 \\
\hline IV & 35 & $38 \%$ & 1 & $1 \%$ & 26 \\
\hline Total & 87 & $94 \%$ & 6 & $6 \%$ & 100 \\
\hline
\end{tabular}


a satisfacción familiar, posteriormente los de rendimiento académico y por último lo encontrado en la correlación satisfacción familiar/rendimiento académico.

Los datos sociodemográficos tienen que ver con género, edad, estrato social, personas con las cuales vive. En cuanto a género, los datos se recogen en la tabla 5. Los datos muestran que la mayoría de los estudiantes son de género femenino, lo cual muestra la tendencia a la feminización de la carrera psicología.

En cuanto a la edad es una población con una edad media de 18,6 años en un rango que va de los 16 a los 28 años. En el promedio de edad no se tuvieron en cuenta dos casos atípicos cuyas edades superaban los 40 años. La distribución por semestres según dos rangos establecidos $16-21$ y $22-28$ se puede observar en la tabla 6.

En relación con la distribución por estrato socioeconómico, obtenido a partir de la percepción de los participantes, la tabla 7 muestra. Los resultados muestran que los estudiantes de Psicología de la UPB proceden en su mayoría de clases económicas media y media baja. Situación que había sido hallada por Villamizar y Romero (2011).

Se consideró importante para la investigación identificar con quienes viven los participantes, en la medida que una de las variables a considerar es la de satisfacción familiar así como la percepción que ellos tienen respecto a la relación con sus padres.
En la tabla 8 se presenta la descripción de las personas con las cuales los participantes viven: Los datos muestran que la mayoría de los estudiantes viven con sus padres, se presume que esto se encuentra relacionado con factores como la edad, la mayoría son adolescentes o jóvenes que no trabajan y por tanto con dependencia económica.

En cuanto a la percepción sobre la calidad de la relación que tienen con sus padres, en la tabla 9 de muestra la distribución de los resultados: Los datos muestran que la mayoría de los participantes consideran la relación que llevan con sus padres como buena, más no excelente, puede llevar a suponer la existencia de conflictos, lo cual es propio del momento que viven unos y otros: hijos que están dejando la adolescencia y padres que empiezan a vislumbrar el desprendimiento de ellos.

Respecto a la variable satisfacción familiar los resultados se pueden observar en la tabla 10. Los resultados aparentemente muestran alta satisfacción familiar por parte de los estudiantes, pero si se desglosa el nivel de satisfacción en dos grupos alta y moderada, lo que se percibe es que la mayoría de los estudiantes están en el segundo grupo, lo que señala ciertas dificultades a nivel de las relaciones familiares. Estos hallazgos coinciden con los encontrados en la percepción del nivel de relación padres-estudiantes, donde manifestaron, en su mayoría, que eran buenas pero no excelentes.

Tabla 7. Distribución de los participantes según estrato socioeconómico.

\begin{tabular}{ccc}
\hline Estrato & Participantes & Porcentaje \\
\hline 1 y 2 & 15 & $16 \%$ \\
3 y 4 & 67 & $70 \%$ \\
5 y 6 & 13 & $14 \%$ \\
\hline
\end{tabular}

Tabla 8. Descripción de las personas con las cuales viven los participantes.

\begin{tabular}{ccc}
\hline Personas con las cuales vive & Participantes & Porcentaje \\
\hline Padres y hermanos. & 70 & $74 \%$ \\
Otros & 25 & $26 \%$ \\
\hline
\end{tabular}

Tabla 9. Percepción de los participantes respecto a la calidad relación con sus padres.

\begin{tabular}{ccc}
\hline Relación & Participantes & Porcentaje \\
\hline Excelente & 43 & $45 \%$ \\
Buena & 47 & $50 \%$ \\
Regular & 5 & $5 \%$ \\
\hline
\end{tabular}


Tabla 10. Nivel de satisfacción de la totalidad de los estudiantes.

\begin{tabular}{ccc}
\hline Nivel de satisfacción & Estudiantes & Porcentaje \\
\hline Muy baja satisfacción familiar & 2 & $2 \%$ \\
Baja satisfacción familiar & 30 & $32 \%$ \\
Moderada satisfacción familiar & 17 & 185 \\
Alta satisfacción familiar & 46 & $48 \%$ \\
Total & 95 & 1005 \\
\hline
\end{tabular}

Tabla 11. Baremos calificativos de la UPB.

\begin{tabular}{cc}
\hline Notas & Criterios \\
\hline $0.0-1.9$ & Malo \\
$2.0-2.9$ & Deficiente \\
$3.0-3.9$ & Regular \\
$4.0-44$ & Bueno \\
$4.5-5.0$ & Excelente \\
\hline
\end{tabular}

Tabla 12. Promedios generales de los estudiantes de II, III y IV semestre.

\begin{tabular}{cccc}
\hline Semestre & II & III & IV \\
\hline Promedio & 3.84 & 3.72 & 3.67 \\
\hline
\end{tabular}

Tabla 13. Relación entre nivel de satisfacción y rendimiento académico.

\begin{tabular}{cc}
\hline Nivel de satisfacción & Rendimiento académico \\
\hline Muy baja satisfacción familiar & 4.0 \\
Baja satisfacción familiar & 3.7 \\
Moderada satisfacción familiar & 3.9 \\
Alta satisfacción familiar & 3.8 \\
Total & 3.8 \\
\hline
\end{tabular}

Tabla 14. Correlación entre nivel de satisfacción familiar y rendimiento académico en la totalidad de participantes.

\begin{tabular}{cc}
\hline $\mathrm{r}$ & Significancia \\
\hline .023 &, 783 \\
\hline
\end{tabular}

Significancia .05

Tabla 15. Rendimiento académico y satisfacción familiar por semestre.

\begin{tabular}{ccc}
\hline Semestre & Nivel de satisfacción & Rendimiento académico \\
\hline \multirow{2}{*}{ II } & Muy baja satisfacción familiar & 4.0 \\
& Baja satisfacción familiar & 3.8 \\
& Moderada satisfacción familiar & 4.1 \\
& Alta satisfacción familiar & 3.8 \\
III & Baja satisfacción familiar & 3.3 \\
& Moderada satisfacción familiar & 3.8 \\
& Alta satisfacción familiar & 3.8 \\
& Muy baja satisfacción familiar & 4.0 \\
& Baja satisfacción familiar & 3.3 \\
& Moderada satisfacción familiar & 3.5 \\
& Alta satisfacción familiar & 3.7 \\
\hline
\end{tabular}


Tabla 16. Correlación entre nivel de satisfacción familiar y rendimiento académico por semestre

\begin{tabular}{ccc}
\hline Semestre & $\mathrm{r}$ & Significancia \\
\hline II & .069 & .558 \\
III & .248 & .216 \\
IV & .246 & .119 \\
\hline
\end{tabular}

Sobre la variable rendimiento académico, la UPB tiene un sistema de calificaciones de tipo cuantitativo, las notas oscilan entre 0.0 y 5.0 , considera que las asignaturas se pierden cuando se sacan menos de 3.0. A nivel cualitativo los investigadores proponen los criterios, que se recogen en la tabla 11.

Teniendo en cuenta que como rendimiento académico se consideró el promedio de las notas obtenidas por los estudiantes durante su permanencia en la UPB, se encontró que el promedio general de los participantes de 3.74 , puntaje que según la tabla dada, es considerado regular. Al parcelar el grupo de acuerdo al nivel de formación, se obtuvieron los resultados que se presentan en la tabla 12. Los resultados muestran que el promedio académico disminuye en la medida que el nivel de formación aumenta, se puede suponer que el nivel de dificultad es mayor en la medida que se avanza en el estudio de la carrera.

De acuerdo a los criterios cualitativos establecidos se considera el rendimiento académico como regular en los tres semestres debido a que es menor de 4.0.

Teniendo en cuenta que la investigación buscó determinar el nivel de relación entre las variables satisfacción familiar y rendimiento académico, inicialmente se realizó tal relación a nivel general, es decir con toda la población, luego por niveles. Los resultados de la primera relación se presentan en la tabla 13.

Para determinar la existencia o no de correlación entre Nivel de Satisfacción y Rendimiento Académica aplicó el $r$ de Pearson el resultado se presenta en la tabla 14. El nivel de significancia encontrado muestra que $r$ no es estadísticamente significativas por tanto se afirma que no existe relación entre satisfacción familiar y rendimiento académico.

Con el fin de dar respuesta a uno de los objetivos específicos se buscó encontrar la relación entre nivel de satisfacción familiar y rendimiento académico por semestre, lo hallado se presenta en la tabla 15. Para determinar las correlaciones entre el rendimiento académico, por semestre, y los resultados arrojados por el ESFA, se aplicaron los respectivos coeficientes de correlación, los resultados encontrados se presentan en la tabla 16. Los resultados muestran que no se presenta correlación entre rendimiento académico y satisfacción familiar en ningún semestre.

\section{DISCUSIÓN}

Los datos muestran que no se presenta una relación directa entre satisfacción familiar y rendimiento académico, por tanto, se puede afirmar que los estudiantes que tienen alto promedio académico no necesariamente perciben alta satisfacción familiar y viceversa.

Estos resultados permiten dar cuenta de lo acertada que es la premisa que habla sobre la intervención de una multiplicidad de variables en el rendimiento académico, factores tanto internos (hábitos y herramientas de estudio; motivación e interés en el estudio) como externos (contexto social y familiar). Consecuente con dicha premisa se encontró que el estar o no satisfecho con el núcleo familiar hace que una persona tenga alto o bajo rendimiento académico.

Los resultados muestran que la percepción sobre satisfacción familiar que tienen los estudiantes es buena, no la visualizan como excelentes, posiblemente porque se presentan problemas entre los diferentes miembros que conforman la familia, situación por lo demás esperada por las características de la población, personas con promedio de edad de 18.6 años y estudiantes universitarios, que están en proceso de desligamiento, además el ambiente universitario y las demandas que a nivel social que allí se dan rompen con las que dentro de las familias se habían establecido, los padres siente que empiezan a perder el control, que su autoridad disminuye, situación que se relaciona con lo encontrado por Cely y Ojeda (2003) donde muestran la percepción de los padres respecto a la relación con sus hijos: un 7\% de ellos comienzan a verlos como independientes, es decir asumen ese cambio, pero qué sucede con el $93 \%$ restantes, puede estar incubándose por esta percepción de pérdida de autoridad, comportamientos entre uno y otro, es decir entre padres e hijos, que lleve a la aparición de 
problemas. En todo caso, los datos no indican que se presente una situación altamente conflictiva en la relación familiar, de forma tal que logre afectar otras áreas de desempeño como la escolar.

En lo que atañe a la relación de las variables satisfacción familiar vs. rendimiento académico, estadísticamente no se presentó relación alguna tanto a nivel general como por semestre. Estos resultados difieren de lo hallado en otras investigaciones sobre la misma temática, por ejemplo la de Martínez (1997) citado por Paz-Navarro, Rodríguez y Martínez (2009) quien halló que el funcionamiento familiar influye en el rendimiento escolar y los reportados por GarcíaCruz, Guzmán y Martínez (2006) quienes encontraron relación entre clima familiar y rendimiento académico.

Los resultados dejan ver que el rendimiento académico de los participantes no es el mejor, y que a medida que avanzan en el proceso de formación el promedio disminuye, lo que permite suponer que el nivel de complejidad también se incrementa y que en el rendimiento median diversos factores, sin desconocer, según las diversas investigaciones lo señalan, que el entorno familiar y lo que allí sucede incide en los logros académicos.

La investigación permitió identificar ciertas características tanto de los estudiantes de Psicología de la UB Bucaramanga como de sus familias:

\section{Características de los estudiantes.}

1. Respecto al género: feminización de la carrera, aspecto explicado por Álvarez-Uría, Varela, Gordo y Parra, citados por Villamizar y Romero (2011) por el tipo de servicio que prestan estos profesionales que es visto como de ayuda.

2. En aspectos socioeconómicos: el nivel social por lo general corresponde a estrato tres, resultados que también coinciden con los autores ÁlvarezUría, Varela, Gordo y Parra, citados por Villamizar y Romero (2011), quienes notaron que el 90\% de los estudiantes españoles de Psicología pertenecen a la clase trabajadora.

3. En relación con la edad: el promedio es de 18.6 años, ingresan a la universidad alrededor de los 16 años.

4. Procedencia: la mayoría proceden del área metropolitana de Bucaramanga, viven con sus padres, de los cuales dependen económicamente, son solteros y pertenecen a familias nucleares.
El vivir con los padres concuerdan con datos sociodemográficos hallados. La población universitaria colombiana según la investigación "Cómo viven los estudiantes hoy", citado por Universia (2007) un $6 \%$ de los estudiantes que ingresan tienen 16 años, el 12\% tiene 17 , un $16 \%$ llega a los 18 años, y sólo el $9 \%$ entra a estudiar después de los 24 años.

\section{Características de las familias.}

1. Tienen tendencia al conservadurismo.

2. Son cerradas, no permiten la intromisión de otros en sus asuntos.

3. Cohesionadas, sus miembros se protegen y ayudan mutuamente.

Es indispensable considerar que los datos son producto de la percepción de los estudiantes, ellos consideran a sus familias de esta manera, estos resultados pueden cambiar al trabajar con otro grupo, razón por la cual no se puede generalizar.

\section{REFERENCIAS BIBLIOGRÁFICAS}

1. Ackerman, N. (1958). Diagnóstico y tratamiento de las relaciones familiares. Psicodinamismos de la vida familiar. Buenos Aires: Hormé.

2. Aldana, A; Pérez, R. \& Rodríguez, A. (2010). Visión del desempeño académico estudiantil en la Universidad Centroccidental Lisandro Alvarado. Compendium.24, 5-21.

3. Andrade, M. \& Montalvo, R. (2006). Problemas de adaptación de un adolescente: estudio de caso bajo modelo estructural. Revista Electrónica de Psicología Iztacala. 9 (2), 84-103.

4. Artunduaga, C (2008). Variables que influyen en el rendimiento académico en la universidad. Recuperado 5 febrero, 2011, de http://www. slideshare.net/1234509876/variables-delrendimiento-acadmico-universidad.

5. Barraca, J. \& López-Yarto, L. (1997). ESFA Escala de satisfacción familiar por adjetivos. Madrid: TEA Ediciones.

6. Cely, C. \& Trouchon, M. (1999). Características personales, familiares y académicas de los estudiantes admitidos al primer nivel en los programas académicos de la UPB 1998. Trabajo no publicado. Departamento General de Investigaciones UPB: Bucaramanga.

7. Cely, C. \& Ojeda, M. (2003). Características personales, familiares, académicas y actitudinales de los estudiantes que ingresaron en el 2001 a la UPB 1998. Trabajo no publicado. Departamento General de Investigaciones UPB: Bucaramanga. 
8. Contreras, K., Caballero, C., Palacio, J. \& Pérez, A. (2008). Factores asociados al fracaso académico en estudiantes universitarios de Barranquilla (Colombia). Psicología desde el Caribe. 22, 110-135.

9. Corominas, J. (2003). Breve diccionario etimológico de la lengua castellana. Madrid: Gredos.

10. Corsi, J. (2003). Maltrato y abuso en el ámbito doméstico. Buenos Aires: Paidós.

11. Edel, R. (2003). El rendimiento académico: concepto, investigaciones y desarrollo. Revista Electrónica Iberoamericana sobre Calidad, Eficacia y Cambio en Educación, 1(2), 1-15.

12. Engels, F. (1976). El origen de la familia, la propiedad privada y el Estado. Moscú: Progreso.

13. Enríquez, C. (2008). Factores de riesgo asociados a bajo rendimiento académico en escolares de dos instituciones educativas públicas de Bogotá. Medellín: Universidad CES.

14. Espinal, I, Gimeno, A \& González, F. (2003). El enfoque sistémico en los estudios sobre la familia. Universidad Autónoma de Santo Domingo UASD y Centro Cultural Poveda. Distrito Nacional Santo Domingo. República Dominicana. Recuperado 8 marzo, 2011, de http://www.uv.es/jugar2/ Enfoque\%20Sistemico.pdf

15. Estupiñan, J. \& Córdoba, A. (1992). Marco conceptual para el estudio de la familia desde una perspectiva sistémica. Aportes a la Psicología. 1 (1), 5-22.

16. Galvis, L. (2001). La familia. Una prioridad olvidada. Bogotá: Aurora.

17. Garbanzo, G. (2007). Factores asociados al Rendimiento Académico en estudiantes universitarios, una reflexión desde la calidad de la Educación Pública. Educación, 31 (1), 43-63.

18. García, C. (2005). Habilidades sociales, clima social familiar y rendimiento académico en estudiantes universitarios. Liberabit.11, 63-74.

19. García-Cruz, R., Guzmán, R. \& Martínez, J. (2006). Tres aristas de un triángulo: bajo rendimiento académico, fracaso y deserción escolar, y un centro: "el adolescente". Revista Cientifica Electrónica de Psicología. 2, 8-33.

20. Guerra, T. (1993). Clima social familiar en adolescentes y su influencia en rendimiento Académico. Trabajo de Grado para optar el título de Psicóloga. Universidad Nacional Mayor de San Marcos. Lima.

21. Hernández, A. (1992). Estructura y funcionamiento de familias colombianas no clínicas según el Modelo Circumplejo de D. Olson. Aportes a la Psicología. 2, 17-27.

22. Hernández,A. \& Echevarría, K. (2007). Características de la estructura familiar de los estudiantes de la facultad de Administración de Empresas, Derecho, Ingeniería Industrial, Ingeniería Civil, Ingeniería Electrónica e Ingeniería Informática de la Universidad Pontificia
Bolivariana seccional Bucaramanga. Trabajo de Grado para optar el título de Administradora de Empresas. Universidad Pontificia Bolivariana. Bucaramanga.

23. Higuera, M. \& Rodríguez, M. (2002). Características actitudinales de los estudiantes admitidos en los diferentes programas académicos de la UPB de Bucaramanga 2001. Trabajo de Grado para optar el título de Psicóloga. Universidad Pontificia Bolivariana: Bucaramanga.

24. Martínez, M. (2011). Programa de Acompañamiento Académico. Recuperado 10 marzo, 2011, de http:// www.upbbga.edu.co/bienestar/bienestaru/pac.html

25. Martínez, A., Sanz, M., \& Cosgaya, L. (2005). El papel de las relaciones familiares en el rendimiento escolar $\mathrm{y}$ en el bienestar psicológico de los adolescentes. Recuperado 7 de Agosto, 2011, de http://psicoteca.blogspot.com.

26. Membrillo, A. \& Ocampo, P. (2008). Teoría general de los sistemas y la familia como sistema. En Familia. Introducción al estudio de sus elementos. A. Membrillo; M. Fernández; J. Quiroz y J. Rodríguez. (Eds.). Familia. Introducción al estudio de sus elementos. México D.F.: Editores Textos Mexicanos.

27. Minuchin, S. (1984). Técnicas en terapia familiar. Barcelona: Paidos.

28. Narváez, E. (1999). La investigación del rendimiento estudiantil. Problemas y Paradigmas. Caracas: Universidad Central de Venezuela.

29. Nizama, M. (2009). La familia en el Derecho Romano y en el ordenamiento normativo actual. Revista de Derecho y Ciencias Políticas. 66 (1-2), 275-294.

30. Orozco, M. \& Velásquez, V. (2010). Caracterización del riesgo familiar en familias de estudiantes del programa de enfermería, de una universidad pública. Hacia la Promoción de la Salud, 15 (1), 78-91.

31. Palomar, M. \& Suarez, E. (1993). El modelo sistémico en el trabajo social familiar: consideraciones teóricas y orientaciones prácticas. Alternativas. 2, 169-184.

32. Parra, J., Gomaris, V. \& Sánchez, M. (2011). El análisis del contexto familiar en la educación. Revista Electrónica Interuniversitaria de Formación del Profesorado, 14 (1), 177-192.

33. Paz-Navarro, L., Rodríguez, P. \& Martínez, M. (2009). Funcionamiento familiar de alumnos con bajo rendimiento escolar y su comparación con un grupo de rendimiento promedio en una preparatoria de la Universidad de Guadalajara. Revista Educación y desarrollo. 10, 5-16.

34. Pérez, I. (2008). Definición de familia. Una visón del Institut Universitary de Salut mental Vidal i Berrauqer. La revue du REDIF. 1, 9-13.

35. Presidencia de la Republica de Colombia. (2010). Constitución Politica de Colombia. Bogotá: Legis. 
36. Reyes, Y. (2003). Relación entre el rendimiento académico, la ansiedad ante los exámenes, los rasgos de personalidad, el autoconcepto y la asertividad en estudiantes de primer año de Psicología de UNMS. Trabajo de Grado para optar el título de Psicóloga. Universidad Nacional Mayor de San Marcos. Lima.

37. Rodríguez, M. (2001). discursos y representaciones familiares sobre género y poder. Trabajo de grado para optar al título de socióloga. Universidad de Chile. Santiago de Chile.

38. Sobrino, L. (2008). Niveles de satisfacción familiar y de comunicación entre padres e hijos. Avances Psicológicos, 16 (1), 109-136.

39. Soria, R. (2010). Tratamiento sistémico en problemas familiares. Análisis de caso. Revista Electrónica de Psicología Iztacala. 13 (3), 87-104.

40. Tinto, V. (1989). Definir la deserción: Una cuestión de perspectiva. ANUIES, 71, 33-51.
41. Torres, L. y Rodríguez, N. (2006). Rendimiento académico y contexto familiar en estudiantes universitarios. Enseñanza e Investigación en Psicología, 11, 255-270.

42. Universia (2007). Cómo viven los estudiantes hoy. Recuperadojunio22,2011,dehttp://noticias.universia. net.co/publicaciones/noticia/2007/10/26/246366/ viven-universitarios-hoy-cifras-reveladoras-estudiosanto-tomas.html

43. Velásquez, D.; Vaiz, R. \& Luiz, J. (2009). Factores de la satisfacción sobre el apoyo familiar del adolescente adicto en tratamiento. Revista Enfermería Herediana. 2 (1):11-19.

44. Villamizar, G. \& Romero, C. (2011). Relación entre variables psicosociales y rendimiento académico en estudiantes de primer semestre de Psicología. Revista Educación y Desarrollo Social. 5 (1), 41-54. 